\title{
Recent Observations of Flickering in CVs and AGN
}

\author{
W. F. Welsh, Janet H. Wood \\ Dept. of Physics, Keele University, Staffordshire, ST5 5BG, UK
}

\author{
Keith Horne \\ School of Physics \& Astronomy, University of St. Andrews, Fife, \\ KY16 9SS, UK
}

\begin{abstract}
Flickering is a phenomenon associated with all known accretion powered processes. Yet despite its energetic importance and universality, our understanding of flickering remains elementary. Only recently has the flickering itself become a topic of serious investigation. This paper briefly addresses two fundamental observational questions: "Where is the flickering coming from?" and "What is the spectrum of the flickering?".
\end{abstract}

\section{Introduction}

"Flickering" is the term used to denote the rapid, apparently random fluctuations seen in the light curves of many astronomical sources (e.g. cataclysmic variables, $\mathrm{X}$-ray binaries, AGN, etc.). There is no preferred time scale and a power spectrum shows a power-law distribution, typically $1 / f$ to $1 / f^{3}$. While the flickering has noise-like characteristics (and often is treated as a noise term), it is important to realize that flickering is intrinsic to the source itself.

Flickering is important for (at least) two reasons: (1) flickering is seen in all accretion-powered sources and is therefore a fundamental signature of the accretion process; (2) the amount of power in the flickering can be a significant fraction of the total luminosity of the source. Yet our understanding of flickering is superficial. This is, to a large extent, due to the lack of observational constraints on the flickering. Recently, there has been an effort to remedy this problem (see, e.g., Bruch 1992, 1996; Welsh, Wood \& Horne 1996).

Two fundamental observational questions about the flickering arise: (1) "Where does the flickering come from?" and (2) "What is the spectrum of the flickering?" To answer the first question, we can use methods of indirect imaging (such as those tomography techniques described in these proceedings by Horne). These include 'eclipse mapping' (for spatial image reconstruction) and 'Doppler tomography' (for velocity field mapping).

To answer the second question, we need, first of all, high-speed spectrophotometry, and secondly, a way to separate the intrinsic variability from the unvarying light and the observational noise. A tool to accomplish this is the simple rms statistic, provided we include a correction term to account for the noise. This "de-biased rms" statistic can be used to measure the amount of intrinsic 
variability in real flux units and therefore can be quantitatively modelled. It is important to stress that this is not a fractional or relative measure, which dilutes the intrinsic variability by sources of light that are unrelated to the flickering (e.g. the secondary star and white dwarf in the case of CVs, or the host galaxy in the case of AGN).

\section{The Location of the Flickering}

From studies of (non-magnetic) eclipsing CVs, we know that the flickering is associated with the accretion disc, central object, and sometimes the hot spot where the gas stream hits the disc rim. Work in progress on eclipse mapping the flickering in the dwarf nova HT Cas has produced an image of the flickering, showing that while the flickering comes from the entire disc it is very concentrated towards the center of the disc (more so than the mean steady light). Further details are discussed in Welsh et al. (1997); also see Bruch (1996) for a thorough investigation of the location of the flickering in the $\mathrm{CV} \mathrm{Z} \mathrm{Cha} \mathrm{(without}$ using eclipse mapping).

\section{The Spectrum of the Flickering}

Using the de-biased rms statistic, the flicker spectrum of several accretionpowered sources has been derived. These include several CVs, a Seyfert galaxy and a BL Lac object. The following general conclusion can be drawn: the spectrum of the flickering can be very different from the mean spectrum of the source, implying that the origin of the flickering is distinct from the origin of the steady, unvarying spectrum. We are not simply seeing changes of brightness; the character of the flickering light is intrinsically different from that of the steady light. In addition, there is considerable evidence that the spectrum of the flickering is timescale-dependent: the fast flickering has a different spectrum from the slow flickering. Any models for the origin of the flickering must take this into account.

\subsection{The CVs TT Ari and HT Cas}

Low resolution data on TT Ari obtained with the HST FOS/RED prism with $3.2 \mathrm{~s}$ time resolution were analysed. The fractional amplitude of the flickering is relatively small, about $4 \%$, as is commonly seen in high-mass transfer CVs (Bruch 1992). The spectrum of the flickering, though crude, reveals a Balmer jump in emission, quite unlike the mean spectrum (Welsh, Wood \& Horne 1996). This result motivated the search for the flickering spectrum in other sources.

HT Cas was observed for $3.6 \mathrm{hr}$ using the HST FOS/G400H with $5.7 \mathrm{~s}$ time resolution. The data cover the wavelength range from $3200 \AA$ to $4800 \AA$, and a strong Balmer jump in emission and double-peaked emission lines are seen. This $\mathrm{CV}$ is faint $(\mathrm{V} \sim 16.5 \mathrm{mag})$, and as a consequence, the count rates are very low, typically $0-4$ counts/pix. To avoid contamination of the rms from orbital motion-induced Doppler shifts of the lines, the data were cast into course wavelength bins. This also gets the count rates out of the Poisson regime and into a regime where the errors on the count rate are Gaussian-distributed. The 
the spectrum of the flickering in HT Cas

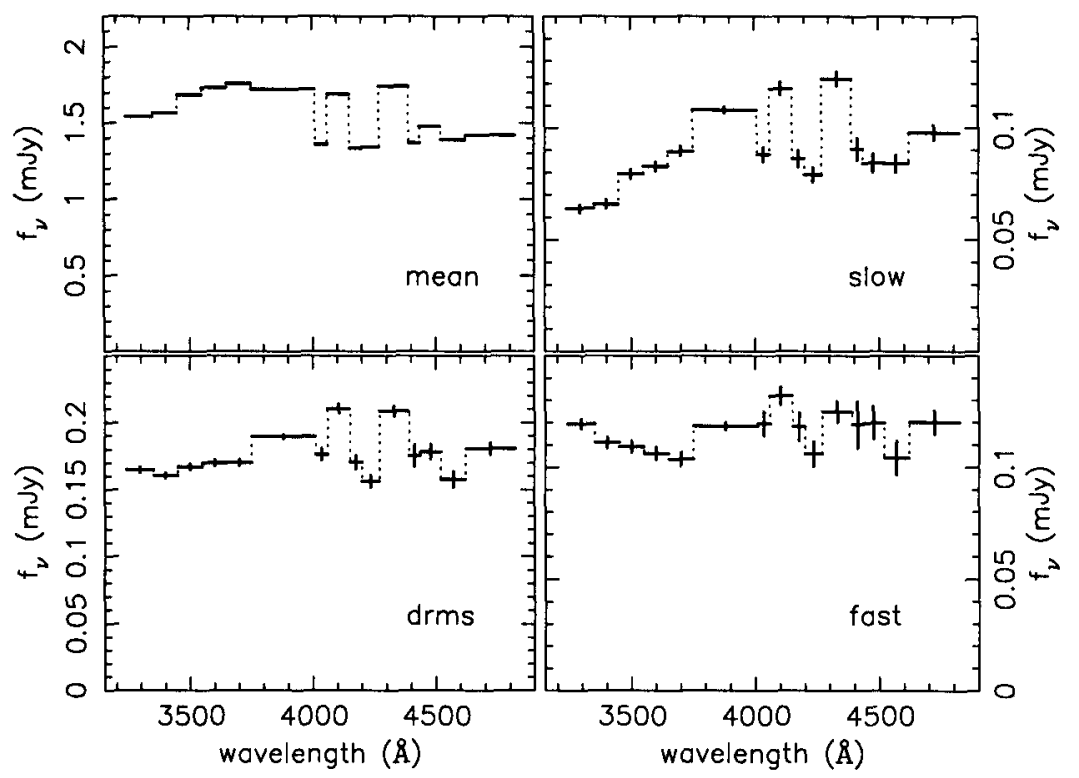

Figure 1. Flickering in HT Cas: mean spectrum, de-biased rms spectrum, spectrum of the slow flickering $(\tau>1 h r)$ and spectrum of the fast flickering $(\tau<30 \mathrm{~min})$.

fractional amplitude of the flickering is 10-15\%, typical of dwarf novae seen at high inclinations. Compared to the mean spectrum, the flicker spectrum shows a less steep Balmer continuum and smaller Balmer jump.

Temporal filtering of the data (to isolate various timescales) reveals the following result: while the slower flickering shows a Balmer continuum rising to the red and a Balmer jump in emission, the faster flickering shows a decreasing Balmer continuum and a Balmer jump in absorption. While an optically thin gas can probably fit the slow flickering, the fast flickering may require either a composite of optically thin plus thick gas with temperature gradient or a very high temperature. In either case, a dramatic change of either optical depth or temperature is needed.

\subsection{The BL Lac PKS 2155-304}

Archival IUE SWP data of this highly variable AGN were examined (see Urry et al. 1993). Unlike the CVs where the fractional amplitude of the variations are typically much less than unity, PKS 2155-304 showed a factor of 2 change in the continuum flux in 30 days. Like most BL Lacs, only a featureless continuum is seen in the spectrum. While the flicker spectrum does not reveal any line features either, the flicker spectrum continuum is significantly bluer than the mean spectrum. 


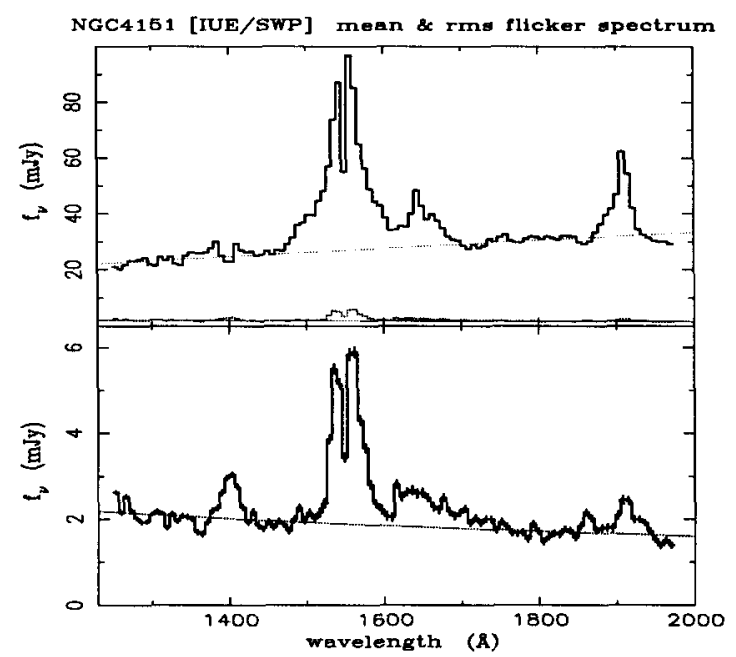

Figure 2. Mean (upper panel) and flickering spectrum of NGC 4151.

\subsection{The Seyfert galaxy NGC 4151}

IUE SWP data (Crenshaw et al. 1996) of this well studied Seyfert I galaxy were examined. However, prior to computing the de--biased rms, the error bars had to be carefully rescaled (the TOMSIPS error bars were far too conservative, i.e., overestimated). A cross-correlation image gave no indication of any time delay between continuum regions (this is also true for HT Cas and PKS 2155-304). Compared with the mean spectrum, the flicker spectrum reveals a much bluer continuum, a much weaker CIII] $1909 \AA$ line, and a much stronger SiIV $1400 \AA$ blend. CIV remained the most prominent spectral feature (Ly $\alpha$ and NV were too contaminated to reliably measure the intrinsic flickering). See also the flicker spectrum shown in Ulrich \& Horne (1996).

\section{References}

Bruch, A. 1992 A\&A, 266, 237

Bruch, A. 1996 A\&A, 312, 97

Crenshaw, D.M., et al. 1996 ApJ, in press

Ulrich, M.-H., \& Horne, K. 1996 MNRAS, in press

Urry, C.M. et al. 1993 ApJ, 411, 614

Welsh, W.F, \& Wood, J.H. 1995 in Greiner, J., Duerbeck, H.W., Gershberg, R.E., eds., Flares 83 Flashes Proc. IAU Coll. 151, Springer-Verlag, Berlin, p. 300

Welsh, W.F, Wood, J.H., \& Horne, K. 1996, in Evans, A., Wood, J.H. eds., Cataclysmic Variables and Related Objects Proc. IAU Coll. 158, Kluwer, in press

Welsh, W.F, Wood, J.H., \& Horne, K. 1997, in progress 